\title{
Addenda: The $h$-index in Australian Astronomy
}

\author{
Kevin A. Pimbblet ${ }^{\mathrm{A}, \mathrm{B}}$ \\ A School of Physics, Monash University, Clayton, VIC 3800, Australia \\ B Email: Kevin.Pimbblet@monash.edu
}

\begin{abstract}
Pimbblet (2011) published an evaluation of the Hirsch $h$-index in the context of the Australian astronomical community. This addenda adds treatment of changes of surname to the computation of the $h$-index and presents derivative data on the $m$-index.
\end{abstract}

Keywords: Errata, Addenda

\section{Change of Surname}

Pimbblet (2011) published a variety of analyses on the $h$-index (e.g. Hirsch 2005) in the context of Australian Astronomy. Alongside that, a discussion of a number of caveats was also made. One further caveat merits explicit attention in the interpretation of the $h$-index: changes of surname. In the Pimbblet (2011) analysis, no attempt was made to track surname changes. This has the effect of depressing the $h$-index of individuals who have changed their names over the years. Its effect can readily be seen in Table 1 where we re-compute the top ten $h$-index for Australian Astronomy and account for changing surnames: Bland-Hawthorn's previous $h$ index was suppressed by 4 points by not taking this in to account. We emphasize that the same dataset was used for this re-computation as the original paper. It is impractical to attempt to account for this for the entire membership of the Astronomical Society of Australia, hence we refrain from publishing percentiles of the $h$ index that account for this correction.

This correction could potentially affect female scientists more than male scientists. We therefore re-issue the caution and caveat emptor that care must be used in the interpretation of any individual's $h$-index and that the tables published by Pimbblet (2011) have deficiencies: both known and unknown.

Finally we note that upon request, ADS is able to create a synonym for surname changes. Once articles with both surnames exist within ADS, a search upon either name will return all results (Grant, priv. comm.).

\section{$2 m$-index}

The data gathered by Pimbblet (2011) can also yield a derived quantity: the $m$-index. The $m$-index is formally the rate of growth of the $h$-index, defined as the $h$-index divided by the number of active research years (Hirsch 2005). Thus a scientist with an $h$-index of 20 and 20 years of active research will have an $m$-index of 1 .

By approximating the number of active research years as the time elapsed since the Ph.D. award date, we are able to obtain an approximation for the $m$-index in Australian Astronomy. The percentiles for the $m$ index distribution for various membership categories
Table 1: Top $10 h$-index for Australian Astronomy, excluding overseas professionals with correction applied for surname changes.

\begin{tabular}{lll}
\hline Rank & Name & $h$ \\
\hline$=1$ & Ken FREEMAN & 77 \\
$=1$ & Jeremy MOULD & 77 \\
3 & Karl GLAZEBROOK & 71 \\
4 & Dick MANCHESTER & 68 \\
5 & Michael DOPITA & 64 \\
6 & Warrick COUCH & 61 \\
7 & Joss BLAND-HAWTHORN & 57 \\
8 & Matthew COLLESS & 56 \\
9 & Brian SCHMIDT & 54 \\
10 & Mike BESSELL & 53 \\
\hline
\end{tabular}

of the Astronomical Society of Australia (see Pimbblet 2011) are presented in Table 2. It is interesting to note how this distribution appears to be shifted toward higher values than generally noted by Hirsch (2005). A careful examination of the individuals at the upper end of the distribution reveals the likely cause of this: many are only one or two years outside of their Ph.D. award date. Therefore, we recompute the $m$-index distribution for individuals who have had a minimum of 5 years of active research time in the last row of Table 2. This provides a much more realistic treatment of the high end of the $m$-index distribution. Moreover, it is also broadly consistent with the views expressed in Hirsch (2005) that $m \sim 1$ denotes a successful scientist, $m \sim 2$ are outstanding, and $m \sim 3$ or higher are truly unique individuals. Again though, we remind the reader that this analysis is at best an approximation, particularly given the issues noted above.

\section{Acknowledgments}

KAP thanks Joss Bland-Hawthorn, Sarah Brough, Martin Asplund, and Andrew Hopkins for discussion, and many other correspondants for encouragement.

This research has made use of NASA's Astrophysics Data System Bibliographic Services. 
Table 2: Percentiles of $m$-index distribution. The sample labelled ' 5 ' are those researchers in M+F-R-O categories who have had five or more years active research as defined by their Ph.D. award date - see text for detail.

\begin{tabular}{rllllllll}
\hline Sample & $\mathrm{N}$ & \multicolumn{8}{c}{ Percentile } \\
& & 25 & 50 & 75 & 90 & 95 & 97.5 & 99 \\
\hline $\mathrm{M}+\mathrm{F}+\mathrm{R}-\mathrm{O}$ & 242 & 0.64 & 1.25 & 1.89 & 2.43 & 3.00 & 4.20 & 4.87 \\
$\mathrm{M}+\mathrm{F}-\mathrm{R}-\mathrm{O}$ & 206 & 0.91 & 1.47 & 2.04 & 2.50 & 3.18 & 4.00 & 4.98 \\
5 & 183 & 0.83 & 1.37 & 1.93 & 2.36 & 2.84 & 3.35 & 4.03 \\
\hline
\end{tabular}

\section{References}

[1] Hirsch, J.E. 2005, Proc. Nat. Acad. Sciences 102, 16569

[2] Pimbblet, K.A. 2011, PASA, 28, 140 